\title{
Lack of significant difference between internal spermatic vein and brachial vein ischemia modified albumin levels in patients with varicocele
}

\author{
Yuksel Aliyazicioglu ${ }^{1}$, Emin Ozbek ${ }^{2}$, Levent Ozcan ${ }^{3}$, S. Sami Cakir ${ }^{2}$, Murat Dursun ${ }^{2}$ \\ ${ }^{1}$ Department of Biochemistry School of Medicine, Karadeniz Technical University, Trabzon, Turkey; \\ ${ }^{2}$ Okmeydani Research and Education Hospital, Department of Urology, Istanbul, Turkey; \\ ${ }^{3}$ Sinop State Hospital, Department of Urology, Sinop-Turkey.
}

\begin{abstract}
ГSummary
Varicocele is the most common and surgically correctible cause of male infertility in men attending to infertility clinics. Infertility affects $15 \%$ of all couples and male factor is the primary or contributing cause in $40 \%$ to $60 \%$ of cases. Varicocele has been shown to cause male infertility in about $15 \%$ of infertile couples. Molecular mechanisms responsible from varicocele induced testicular dysfunction and male infertility have not been completely unknown. Recent years have witnessed a huge amount of scientific works devoted to the mechanism of varicocele associated male infertility and rapid progress in research on its cellular and molecular mechanisms, including apoptosis and oxidative stress of germ cells. Here we evaluated internal spermatic vein and brachal vein ischemia modified albumin (IMA) level in 40 adult male patients with varicocele. IMA level was analyzed using albumin cobalt-binding test. Spermatic vein and brachial vein IMA levels were $0.381 \pm 0.135$ ABSU (absorbance units) and $0.385 \pm 0.131 \mathrm{ABSU}$, respectively. There was no statistically significant difference between the two areas. IMA levels in the internal spermatic vein of patients with varicocele should not be used as a marker of hypoxia.
\end{abstract}

KEY WORDS: Infertility; Ischemia modified albumin; Oxidative stress; Varicocele.

\begin{abstract}
INTRODUCTION
Molecular mechanisms responsible from varicocele induced testicular dysfunction and male infertility have not been completely unknown. Recent years have witnessed a huge amount of scientific works devoted to the mechanism of varicocele associated male infertility and rapid progress in research on its cellular and molecular mechanisms, including apoptosis and oxidative stress of germ cells. Here we evaluated internal spermatic vein and brachal vein ischemia modified albumin (IMA) level in 40 adult male patients with varicocele.
\end{abstract}

\section{Materials AND Methods}

After ethical comittee approval a total of 40 normal gonadotropic patients, aged between 23 and 37 years, attending a male infertility clinic because of prolonged (more than 1 years) subfertility were included in the study. All patients were evaluated by physical examination and scrotal color Doppler ultrasonography. All had unilateral grade II or III primary varicocele but no testicular atrophy was diagnosed. On the right side, scrotal Doppler ultrasonography and physical examination were normal in all cases. Those with abnormal hormone analysis, testicular atrophy, genito-urinary tract infection, azoospermia were excluded. The mean sperm concentration was $9.9 \pm 2.3 \times 106$ spermatozoa $/ \mathrm{ml}$, while the mean percentage of motile spermatozoa after $1 \mathrm{~h}$ was $38.1 \pm 1.6 \%$. The mean percentage of morphologically normal spermatozoa was $29.3+0.8 \%$. 
Whole blood samples $(1.5 \mathrm{ml})$ were drawn using a 25 gauge-needle during surgery for varicocele from a brachial vein and from the internal spermatic vein. The dilated spermatic vein was punctured immediately after exposing the spermatic cord and before any further manipulation.

After obtaining blood samples in plain tubes contained separation gels, the samples was allowed to clot for 30 minutes and centrifuged before separating the serum. The samples were then immediately frozen and stored at $-80^{\circ} \mathrm{C}$ for assays IMA. IMA level was analyzed using albumin cobalt-binding test (6).

\section{RESULTS}

Spermatic vein and brachial vein IMA concentrations were $0.381 \pm 0.135$ ABSU (absorbance units) and $0.385 \pm$ 0.131 ABSU, respectively. There was no statistically different at comparison with Mann Whitney U test ( $p>0.05)$.

\section{Discussion}

In a study (1) using expression of the alpha subunit of hypoxia-inducible factor 1 (HIF-1 alpha) as a marker of hypoxia, it was reported that left-sided experimental varicocele in the rat causes bilateral testicular hypoxia and germ cell apoptosis.

Role of nitric oxide (NO) and reactive oxygene species in varicocele induced male infertility have also been extensively reported $(2,3)$.

Ischemia modified albumin (IMA) is produced when hypoxic heart tissue induces modification of circulating albumin (4). Therefore IMA is proposed as a biomarker of ischemia and oxidative stress. Modifications that alter the binding capacity of albumin for cobalt may arise during ischemia as a result of acidosis, reduced oxygen tension and generation of free radicals. Several clinical conditions such as pulmonary embolism, mesenteric ischemia, peripheral arterial occlusion, deep venous thrombosis, stroke and myocardial infarction and testicular torsion are associated with increased serum IMA concentrations (5). Taking into account the evident ischemia-related oxidative stress in varicocele we compared in spermatic and brachial veins of patients with varicocele the IMA level as sensitive marker of ischemia and oxidative stress.

Despite the increased oxidative stress and ischemia related to varicocele, IMA concentrations in spermatic veins were not significantly higher than brachial veins. This finding is difficult to explain, although it may be possibly explained by the presence of only relatively small ischemic areas and by the short transit time of ischemic blood within the dilated internal spermatic veins. As a result of our study we have to conclude that IMA levels in the internal spermatic veins of varicocele patients should not be used as a marker of hypoxia.

\section{REFERENCES}

1. Sun Y, Wang L, Xu C, et al. ypoxia-induced apoptosis in the bilateral testes of rats with left-sided varicocele: a new way to think about the varicocele. J Androl. 2010; 31:299-305.

2. Ozbek E, Turkoz Y, Gokdeniz R, et al. Increased nitric oxide production in the spermatic vein of patients with varicocele. Eur Urol. 2000; 37:172-5.

3. Chen SS, Huang WJ, Chang LS, Wei YH. 8-Hydroxy-2'deoxyguanosine in leukocyte DNA of spermatic vein as a biomarker of oxidative stress in patients with varicocele. J Urol. 2004; 172:1418-21.

4. Kutlu O, Mentese A, Turkmen S, et al. Investigation of the possibility of using ischemia-modified albumin in testicular torsion: an experimental. Fertil Steril. 2011; 95:1333-7.

5. Mastella AK, Moresco RN, da Silva DB, et al. Evaluation of ischemia-modified albumin in myocardial infarction and prostatic diseases. Biomed Pharmacother. 2009; 63:762-6.

6. Bar-Or D, Lau E, Winkler JV. A novel assay for cobalt-albumin binding and its potential as a marker for myocardial ischemia-a preliminary report. J Emerg Med. 2000; 19:311-315.

\section{Correspondence}

Yuksel Aliyazicioglu, MD

Department of Biochemistry School of Medicine-Karadeniz Technical University, Trabzon, Turkey

Emin Ozbek, MD (Correspoinding Author)

Department of Urology - Okmeydani Research and Education Hospital Darul aceze caddesi, 34384, Sisli, Istanbul, Turkey

ozbekemin@hotmail.com

Levent Ozcam, MD

Department of Urology - Sinop State Hospital

Sinop, Turkey

S. Sami Cakir, MD

Murat Dursun, MD

Department of Urology - Okmeydani Research and Education Hospital Istanbul, Turkey 